\title{
MULTIPLE BONE LESIONS CAUSED BY AVIAN-BATTEY MYCOBACTERIA
}

\author{
Report of a Case
}

\author{
Wray Ellis, Sedgefield, Stockton on Tees, England
}

\begin{abstract}
A boy aged fourteen years presented in May 1963 with a left axillary abscess. This had developed seven weeks before, four days after his left hand had been bitten by a pet rabbit, which was kept in an enclosure with hens. The abscess was incised but the pus was not cultured. In December 1963 he was seen by a paediatrician because the abscess was still draining, and there was a complaint of backache. It was noted that he had been given B.C.G. at school in December 1962 because of a negative Mantoux test. Radiographs of the lumbar spine showed no abnormality. After a period of rest in bed the abscess healed, the backache settled, and he appeared to be in good health. In April 1965 he returned with a stiff, painful back. On admission, radiographs showed destructive changes affecting the third and fourth lumbar vertebrae (Fig. 1) and the right sacro-iliac joint. He was given streptomycin and sodium aminosalicylate and nursed on a plaster bed. In July, through a left lateral approach a large abscess was evacuated, necrotic bone excised, and an iliac bone graft inserted. Culture yielded Mycobacterium tuberculosis partially sensitive to streptomycin, viomycin and cycloserine, but resistant to sodium aminosalicylate and isoniazid.

In April 1966 he went home wearing a posterior spinal support and remained well until December 1966 when he developed swellings of the scalp, lower jaw at its angle, and right clavicle. Radiographs showed lesions in all these sites (Figs. 2 to 4). Treatment was by a further course of streptomycin, and surgical excision. Four months later the lesions had all healed. During 1968 he was apparently in good health, but in January 1969 cold abscesses developed in the right iliac fossa and in the left lumbar region. After starting streptomycin and pyrazinamide therapy, the lumbar spine was explored, affected bone removed and a cancellous bone graft inserted from the second to the fourth lumbar vertebrae. After operation the blood uric acid rose to 12 milligrams per cent, presumably from the pyrazinamide; treatment with alka-butazolidine and allopurinol was given.

The organism now isolated by Dr J. B. Selkon of the Public Health Laboratory, Newcastle upon Tyne, was found to be an Avian type of Mycobacterium. Dr J. Marks of the Tuberculosis Reference Library identified the strain as Battey Serotype IV, which was confirmed by Dr W. B. Schaefer of Denver, Colorado, U.S.A. Sensitivity was now limited to cycloserine, and partially to clofazimine (Lamprene). He was receiving cycloserine, ethionamide and rifampicin (Rimactane), and was apparently well until October 1969 when a cold abscess developed in the right groin. A radiograph (Fig. 5) showed a lesion of the right pubic bone, which was curetted. Histological section (Figs. 6 and 7) showed typical tuberculous granulation tissue. In April 1970 he had an acute respiratory infection and an abscess appeared on the right chest wall. Initially haemophilus influenzae was grown from the sputum, but a little later it grew the acid-fast organism. Radiographs showed lung involvement and lesions of the bodies of the sixth, seventh and eighth thoracic vertebrae. It was noticed incidentally that the right clavicular lesion had healed. Treatment was by clofazimine (Lamprene), to which the organism was partially sensitive, and blood transfusions. By December 1970 the sputum was negative but the bone lesion had not improved. In February 1971 costo-transversectomy was done, necrotic bone removed and an iliac graft inserted. After the previous operations the patient's general condition had quickly improved, but this time recovery was slow and the wound did not heal completely. The organism was now resistant to cycloserine, which was stopped. There was partial sensitivity to gentamycin, which was given in 80-milligram doses daily.
\end{abstract}




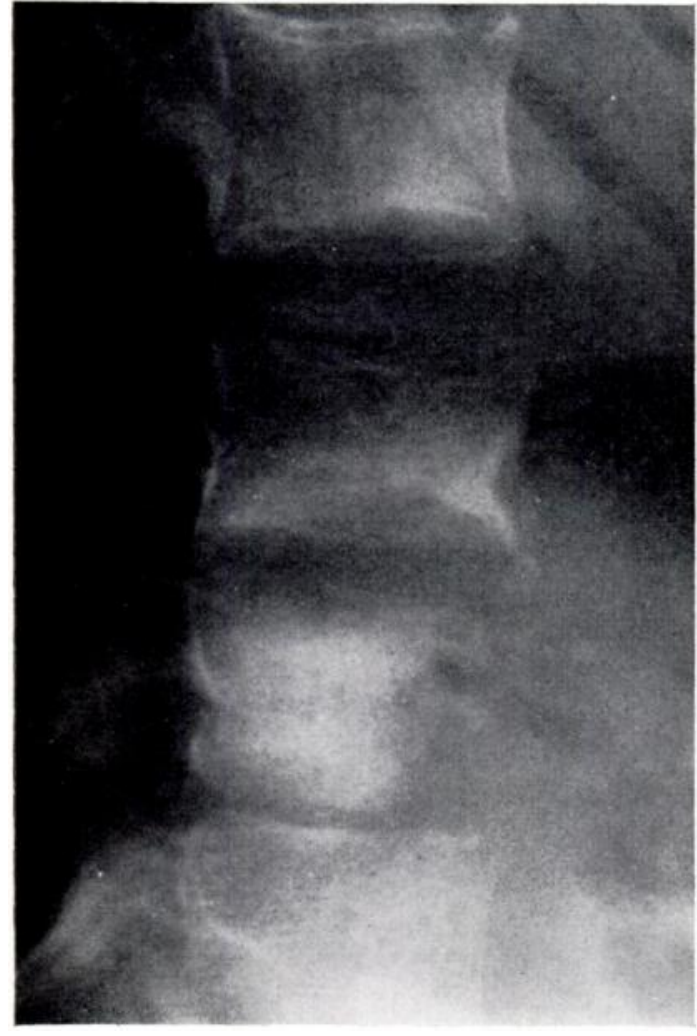

Fig. 1

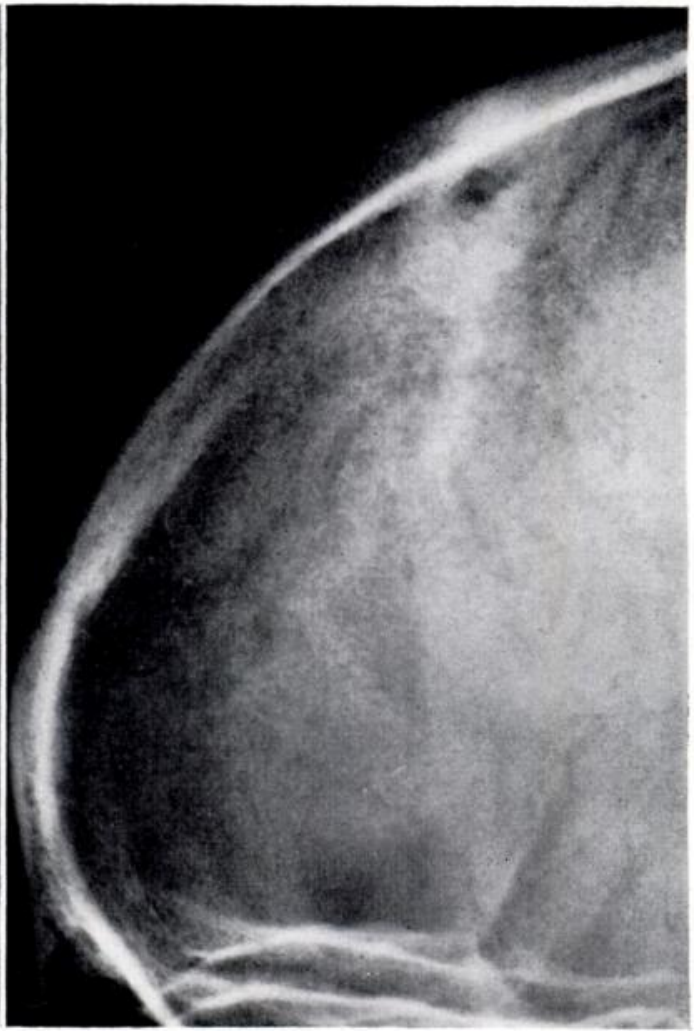

FIG. 2

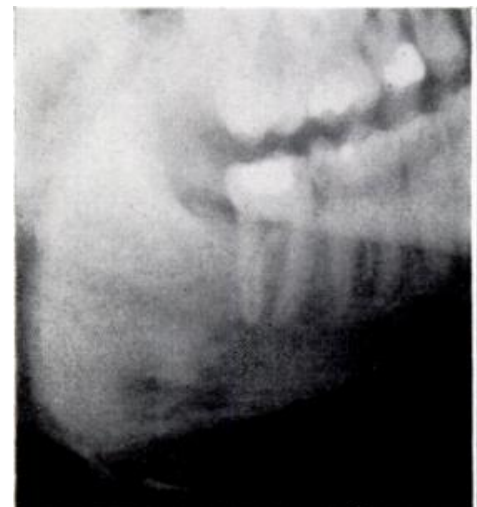

FIG. 3

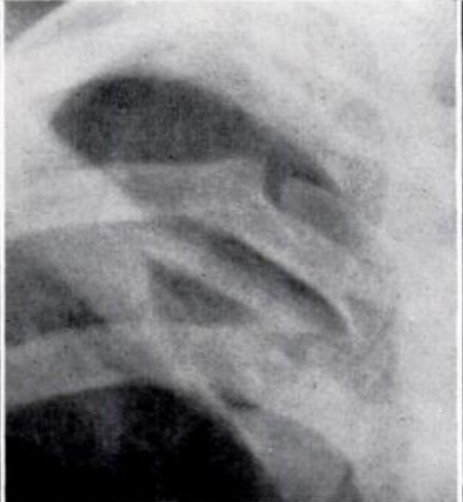

FIG. 4

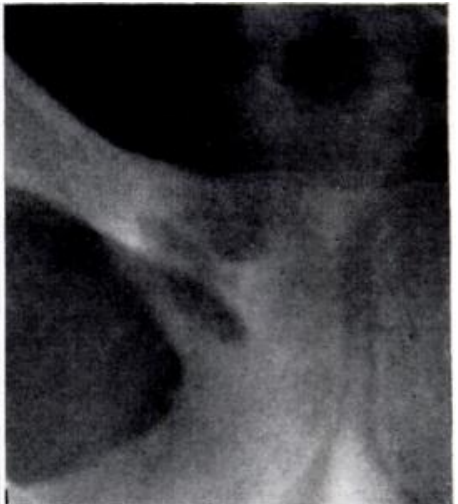

Fig. 5

Figures 1 to 5-Radiographs showing the various lesions. Figure 1 shows destruction of the bodies of the third and fourth lumbar vertebrae. Figures 2 to 5 respectively show the lesions at the junction of the frontal and parietal bones; at the angle of the jaw; in the right clavicle at its inner end; and in the right pubic bone.

He gradually became emaciated, and in October 1971 deafness occurred, presumably from the gentamycin. The drug was stopped, but without relief of the deafness. Clofazimine, the only remaining drug with any laboratory sensitivity, was continued. In January 1972 he developed paraplegia and eight days later died.

At necropsy the larynx, trachea and bronchi were normal. The right lung, adherent to the chest wall, showed numerous areas of fibrosis with two or three small bronchiectatic cavities in the upper lobe. The left lung was covered by pleura five millimetres thick. There 


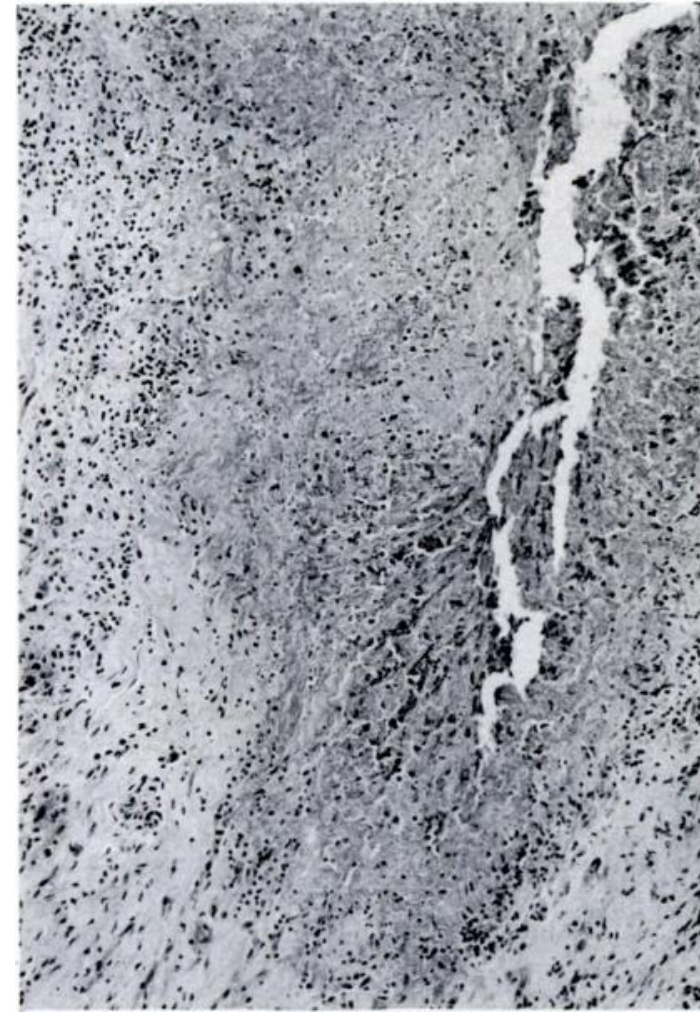

Fig. 6

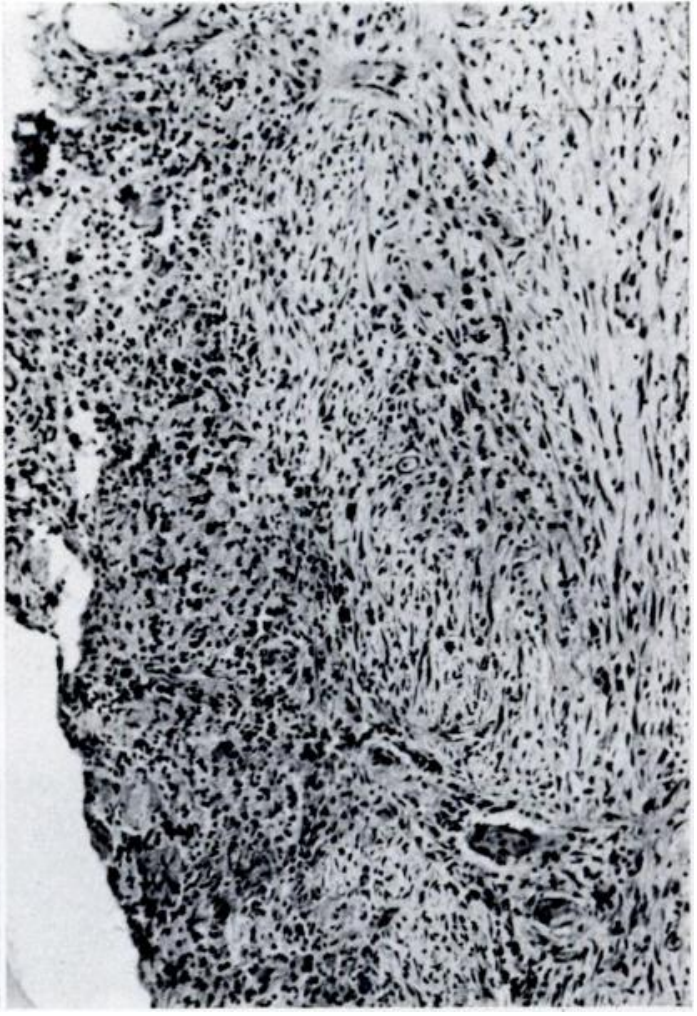

FIG. 7

Figure 6-Histological section of material from the right pubic bone showing the edge of an area of caseous necrosis with a scanty peripheral lymphocytic infiltrate. (Haematoxylin and eosin, $\times 125$.) Figure 7 -Tuberculous granulation tissue from the pubic bone showing a single large multinucleate giant cell. (Haematoxylin and eosin, $\times 125$.)

were a few areas of apparent bronchopneumonia but there was no obvious tuberculosis. The thoracic lymph nodes were normal. There was marked kyphosis where the sixth thoracic vertebra was largely replaced by a mass of thick yellow pus and granulation tissue. The liver showed marked fatty changes. The spleen, enlarged to 500 grams, had amyloid changes. The kidneys were normal.

\section{COMMENT}

Acid-fast organisms of the Avian-Battey strain are often present in soil. The distribution of infection is mainly rural, wild or domestic birds being the natural hosts. There is normally a low pathogenicity for man, and usually an association with pneumoconiosis. Infection of synovial sheaths of the hand, wrist and forearm has been seen in America (Kelly, Karlson, Weed and Lipscomb 1967). Just over a quarter of the cases reported in England and Wales in 1969 were non-pulmonary, mostly being cervical adenitis in children, and none with bone or joint lesions (Schaefer, Birn, Jenkins and Marks 1969). Disseminated infection with Mycobacterium Avium has been reported once in Great Britain (Clark, Thomson and Wallace 1968; Schonell, Crofton, Stuart and Wallace 1968): a girl of twelve presented with mesenteric adenitis, and died four and a half years later after developing multiple bone abscesses, skin sinuses, hepato-splenomegaly, lymphadenopathy and a lung lesion.

The rabbit's bite in a chicken pen probably caused this boy's infection, to which seemingly he had no resistance. He remained Heaf-negative, indicating a lack of tissue response to B.C.G. and to this organism. Early success from surgical treatment evidently depended largely on the antibiotics, because as sensitivity changed and resistance increased his general condition deteriorated. A study of his tissue response was to have been carried out by Professor J. F. 
Soothill of the Institute of Child Health, but the patient became too ill for travel. Although this type of infection is rare, there is a real increase in its incidence (Schaefer et al. 1969), probably in highly susceptible patients who have been spared by the recession of tuberculosis. It might well be advisable in a future case, despite an early favourable response to treatment, to direct the patient to a suitable centre for tissue studies, preferably before blood transfusions have been given. The outcome of these tests, and the development of more suitable antibiotics, appear to be the main hope of combating this still lethal infection.

\section{SUMMARY}

1. A case of multiple bone lesions in a patient infected by Avian-Battey bacillus is reported. 2. It is suggested that the prognosis in future Heaf-negative cases may be improved by studies of the cellular response to the infection.

My thanks are due to my colleagues in the Hartlepool and North Tees groups of hospitals, to Dr J. B. Selkon and Dr A. Wallace of the Public Health Laboratory Service, and to Professor J. F. Soothill of the Institute of Child Health, for their help in the care of this patient, and to Dr R. Lawler for the necropsy findings.

\section{REFERENCES}

Clark, J., Thomson, D. S., and Wallace, A. (1968): Disseminated infection with Mycobacterium avium: Part II-Bacteriology and drug susceptibility. Tubercle, 49, 31-37.

Kelly, P. J., Karlson, A. G., Weed, L. A., and Lipscomb, P. R. (1967): Infection of synovial tissues by mycobacteria other than Mycobacterium tuberculosis. Journal of Bone and Joint Surgery, 49-A, 1521-1530.

Schaefer, W. B., Birn, H. J., Jenkins, P. A., and Marks, J. (1969): Infection with the Avian-Battey group of mycobacteria in England and Wales. British Medical Journal, 2, 412-415.

Schonell, M. E., Crofton, J. W., Stuart, A. E., and Wallace, A. (1968): Disseminated infection with Mycobacterium avium: Part I-Clinical features, treatment and pathology. Tubercle, 49, 12-30. 